\title{
¿Es válida la demostración racional de la existencia de Dios a través de las vías?
}

En el concilio Vat. I se definió que la luz natural de la razón humana puede llegar, partiendo de las creaturas, a conocer con seguridad a Dios como (principio y fin de las cosas) ${ }^{1}$. Según los teólogos, en este texto se habla de la posibilidad de (conocer", no de (probar) o de (demostrar» la existencia de ese principio de todas las cosas; además, el que sea (creador) no formaría parte de la definición ${ }^{2}$. Con esta observación por delante, y ya que las pruebas tradicionales de la existencia de Dios son un modo de conocerle, pero no el único, y pretendiendo éstas ser verdaderas demostraciones y no simple conocimiento, en nada se opondría a la definición del Vat. I quien se pusiera aun en el peor de los casos respecto de las vías, o sea, en el caso de negarles un valor de demostración rigurosa de la existencia de Dios.

Aparte de esto, la demostración racional contenida en las vías sería una entre otras demostraciones de la existencia de Dios, lo que equivale a que las dificultades que puedan suscitarse contra aquéllas pudieran no ser eficaces contra las demás. Si un día se le pidió a Cristo una "mostración) de Dios, al fin y al cabo una especie de prueba, su contestación fue que le mirasen a $\mathrm{El}$, ya que quien le conociese a El conocería también al Padre. Por otra parte, las vías pretenden ser una demostración filosóficamente rigurosa de un Dios trascendente, el de la Biblia, el creador, el "hoc dicimus Deum)", extremo con el que como dijimos antes, el concilio parece no meterse. La distinción entre "principio y fin de las cosas" y (trascendente como personal y creador»)

\footnotetext{
1. "Eadem sancta mater Ecclesia tenet et docet, Deum, rerum omnlum princip' um et finem, naturali humanae rationis lumine e rebus creatis certo cognosci posse". "Si quis dixerit, Deum unum et verum, creatorem et Dominum nostrum... A.S." en DS, 30043026.

2. J. GUITTON, Sentido y valor del ateísmo contemporáneo, (Mundo moderno y noclón do 09), Valencia 1966 , p. 134 ss.

3. S. Th., 1, 23.
} 
hay que hacerla, pues, según se piense de una o de otra manera, así se podría decir que la existencia de Dios habría sido casi universalmente admitida en la historia del pensamiento ${ }^{4}$ o que, por el contrario, el ateísmo habría estadio a la orden del día en todos los tiempos. Al plantear aquí el problema que encabeza estas líneas nos referimos a la demostración filosófica de un principio supremo trascendente en sentid. pleno, como no lo sería, por ejemplo, el dios dialéctico de Hegel.

POSTURAS ANTE EL PROBLEMA

En torno al problema ha habido y sigue habiendo para todos 1: gustos, y la bibliografía sobre él es más que sobreabundante. Así como en teología se nos ha hablado de la (muerte de Dios) como de la cosa más natural, hasta el punto de convertirse algunos teólogos en asertores de dicha teoría como si ella fuese la última palabra del pensamiento cristiano, aireando lo que ellos llaman un (ateísmo cristiano" ${ }^{5}$, así también en filosofía hay filósofos, aun entre los católicos, que, con un radicalismo absoluto, no dudan en afirmar que no existen ni pueden existir pruebas de la existencia de Dios ${ }^{6}$. Pero, chasta qué punto es verdad que Dios ha muerto también en el ámbito de la filosofía? ¿En qué sentido el Dios de la filosofía puede ser un Dios contrahecho? $¿$ ¿Hasta qué punto la razón ayudada por la revelación podrá encontrarse con un Dios auténtico, siempre, claro está, dentro de un plano puramente filosófico? Limitándonos a las pruebas tradicionales veremos luego lo que nos parece se deba responder a estas preguntas. Desde luego, el tema no parece fácil cuando, como Hirschberger confiesa en el artículo citado, los mismos defensores de la validez de las vías, se encuentran con no pequeñas dificultades. El, por su parte, cree que dichas pruebas no merecen ser tratadas tan mal y, consiguientemente, intenta una justificación de las mismas en el mismo Aristóteles. Algunos otros piensan, refiriéndose a las vías tomistas, que, con algunos (arreglillos), podrían pasar. Por mi parte creo, sin embargo, que si los (arreglillos), se refieren solamente a una formulación no con "la concisión con que el Santo dibuja las cinco vías que han convertido en

\footnotetext{
4. OLIVIER A. RABUT, Le problème de Dieu Inscrit dan l'évolution, éd. du CERF 1963.

5. ¿Ateismo, cristiano? en la Civitá Católica, set. 1970, pp. 345-350.

6. COLIN $P$, Le théisme et les preuves classiquass de l'existence de Dieu, en L'existence de Dieu, Casterman 1963, p. 135 ss. HIRSCHBERGER, J., Pruebas de la existencla de Dios, en Hombre, Dios y Revelación, ed. SIGUEME, Salamanca 1968, p. 101.
} 
esqueletos los raciocinios primigenios provenientes de la antigüedad") ", esto sería demasiado poco para obviar las auténticas dificultades en contra. Es cierto que el hecho de que dichas pruebas sean tan antiguas en la historia como el mismo pensamiento filosófico aconseja cautela para no sentirnos sin más con derecho a descalificarlas. $Y$ también es cierto que no debe ocultársenos el peligro que significa para la misma fe en la existencia de Dios el privar a la razón humana de alguna posibilidad de una demostración racional de esa existencia de Dios. Como muy bien advierte Hirschberger, (un día aparecerá la pregunta sobre la verdad de la idea de Dios, y si entonces no se puede fundamentar de manera sólida, se desmoronará lo que hasta entonces era fe viva ${ }^{8}$; en este caso seríamos nosotros de mucho menos aviso que el mismo Kant quien, si bien llegó a la conclusión de que la razón no podía demostrar la existencia de Dios, creyó, sin embargo, que con su sistema filosófico, juzgado como auténtico, imposibilitaba al adversario que, con razones también, intentara negarla, según su famosa frase: "tuve que anular el saber para reservar un sitio para la $\mathrm{fe}^{9}$. Precisamente por esto, y porque la verdad nunca dañará a la verdad, procuraremos exponer en estas páginas lo que creemos ser una auténtica dificultad contra el valor probativo de las pruebas tradicionales, sin que por lo mismo, terminemos por echarlas al cesto, ni tampoco por esbozar una sonrisa de conmiseración como suelen hacerlo quienes simplemente han oído campanas.

\section{DificulTADES Y DIFICULTAD}

Cuando se ha abordado el tema acerca de la validez de las vías para una demostración de la existencia de Dios, se ha aludido a ciertas objeciones o dificultades contra las mismas que, a mi modo de ver, no llegan a lo sustancial del problema, a lo que tiene de verdaderamente dificultoso. Así, por ejemplo, siguiendo a Hirschberger en el libro citado, enumeramos las siguientes: a) la demostración racional nos da como resultado un Dios diferente del de la Biblia. Hirschberger responde bien a esta objeción y a ella nos remitimos ${ }^{10}$; me bastará citar lo que me parece más expeditivo de la respuesta: (el que esgrime dicha objeción no sabe en realidad de qué se trata). Por nuestra parte añadi-

7. HIRSCHBEFGER, J., o. c., p. 140.

8. Ib., p. 102.

9. KANT, E., Crítica de la Razón pura (Prólogo). 
ríamos: ¿acaso al Dios de Abraham y de Isaac no le conviene objetivamente la noción filosófica de la aseidad, atributo general como término de cada una de las vías?; aunque, naturalmente, el Dios de $\mathrm{A}$ braham incluya muchísimas otras perfecciones. No va por ahí la dificultad. De todos modos sí distinguiremos entre una demostración puramente racional de la existencia de Dios y la demostración racional de Sto. Tomás que se mueve en la luz de una cierta precomprensión de

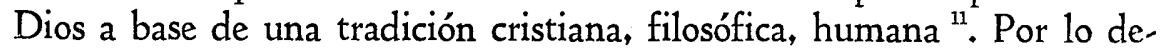
más, ya el mismo Santo nos advierte que, filosóficamente, acerca de Dios más bien sabemos lo que no es que lo que es», siguiendo en esto a S. Agustín ${ }^{12}$. b) Una segunda dificultad podría ser ésta : la demostración metafísica de la existencia de Dios es un esfuerzo inútil porque está formulada según una forma de pensar calejada de la forma ordinaria de pensar de los hombres) ${ }^{13}$. Evidentemente, esto no es una objeción seria; indicaría solamente algo inútil para la mayoría de los hombres no preparados, como no lo está esa mayoría, para las disquisiciones metafísicas. En cambio si la inutilidad se fundamenta en el hecho de que ni a los filósofos modernos convencen, a no ser a aquellos que poseen una fe práctica-existencial en Dios (Max Scheller) y que, por consiguiente, no tienen ya necesidad de pruebas (Marcel), esto ya es más digno de atención. Afirman éstos la imposibidad por parte de la metafísica para consolidar la $\mathrm{fe}$, porque la $\mathrm{fe}$ es radicalmente diferente de la filosofía. Pero la respuesta no es difícil. En primer lugar es cierto que quien posee a Dios por la fe no tiene necesidad de pruebas racionales, pero de ahí no se sigue que éstas sean inútiles, a no ser que se identifique la aceptación de la existencia de Dios por parte de la m. zón que la demuestre con un acto de fe que tenga por objeto esa misma existencia. En este caso, evidentemente, no hay pruebas filosóficas que valgan: la fe, o el acto de fe, no será nunca conclusión de unas simples premisas lógicas. En cualquier otro caso hay que decir que todo lo que la razón pueda descubrir acerca de la verdad será siempre una consolidación de la verdad revelada cuando su descubrimiento sea th. cho sobre una verdad objeto también de la revelación. $\mathrm{Si}$, por ejemplc, la razón demuestra la espiritualidad del alma, la fe en esta verdad quedará consolidada ab extrinseco y por lo mismo será de más fácil acen-

\footnotetext{
11. NICOLETTI, E., La struttura delle cinque vle di S. Tommaso, "Aquinas", I (1969), pp. 57-58. V. Riv. di fil. Neo-Scolastica. mag. giugnn. 1970 , p. 289.

12. In Ps. 85 PL. 37, 1090: "Hoc solum potui dicere, quid non sit".

13. PASCAL, Pensamientos, 543.
} 
tación para aquel que ya la tenga por la razón. Pero lo que realmente sucede es que la demostración metafísica con un punto de partida cosmológico - como es el de las vías - nada puede decir al hombre que está situado filosóficamente en una postura puramante existencialista, y esto por dos motivos: primero porque dichas demostraciones racionales funcionan en un terreno existencial, sí, pero atendidas las relaciones esenciales metafísicas que se dan entre las realidades existentes; pero es aquí precisamente donde el puro existencialista se ha cortado la coleta, ya que, fuera del hombre, sólo se atiende al fenómeno existencial en el que la contingencia de los sucesos fenoménicos tiene sus explicaciones adecuadas dentro del conjunto de la naturaleza y a base de una ciencia física desconocida por los antiguos. El ser en cuanto tal se les ha escapado, - quizás por culpa del idealismo esencialista absorbente de toda realidad contra el que el existencialismo fue una reacción muy justa-, y, por consiguiente, no ven la necesidad de la explicación de una contingencia radical-esencial que les es desconocida. Téngase en cuenta, además, que en el existencialismo, y en general en la filosofía de hoy, las cosas que no son el hombre solamente existen en rèlación con el hombre que es el único que existe auténticamente y que constituye el punto de partida con exigencias de explicación. Es decir, el punto de partida no es ya cosmológico sino antropológico, y el punto de llegada ha de responder más bien a exigencias humanas que del orden físico-existencial. Así, pues, las objeciones presentadas desde este ángulo de visión existencialista responden a una postura filosófica determinada, y en tanto serán válidas en cuanto que la postura filosófica sea la única verdadera. No afectan a los argumentos cosmológicos-metafísicos en sí mismos con una demostración racional en el mismo campo esencial-existencial en el que de hecho y de iure están concebidos aquéllos; en consecuencia no se trata de verdaderas dificultades esenciales a las vías. c) Hay otra serie de dificultades que se refieren a detalles de esta o de aquella vía en concreto, por ejemplo si se trata de un orden físico o no, si de una subordi. nación esencial de las causas o no, o del sentido que puedan tener tales o cuales expresiones; tampoco estas objeciones son auténticas cnmo insuperables, $y$ han sido suficientemente estudiadas y resueltas ${ }^{14}$.

14. CHARLIER, L., Les cinq. voles de Salnt Thomas. Leur structure métaphysique, en L'existance de Diru, pp. 191-227. 


\section{LA AUTENTICA DIFICULTAD}

Cualquiera sabe por la historia de la filosofía que los filósofos han sido capaces de llegar no sólo a la concepción de un ser supremo primordial, sino de superar también un panteísmo de tipo materialista. Ya Anaxágoras se mereció el apelativo, por parte de Aristóteles, de hombre (sobrio entre borrachos) por su descubierta del NOUS ordenador del mundo material y que, a su vez, no es materia ${ }^{15}$. Ahí tenemos también el mundo ideal de Platón, realidad auténtica frente al mundo de la opinión; y sobre todo a Aristóteles ${ }^{16}$. el cual, si bien en el lib. VIII de la Física nos presenta al motor inmóvil como algo inmanente que es pura fuerza física, causa eficiente del movimiento existente en el mundo y con contacto físico con él, sin embargo, en el lib. XII de la Metafísica nos ofrece un concepto de ese ser supremo que supera en absoluto al de la Física, si es que, según otros, pudiera existir incompatibilidad entre ellos ${ }^{17}$. El Motor inmóvil de la Metafísica es (ousia), es (energeia) que mueve no de un modo físico sino como objeto de pensamiento y de deseo; es un ser al que aun la providencia se le discute a fin, precisamente, de colocarlo en la trascendencia del Acto pirro respecto de la movilidad de los demás seres ${ }^{18}$. No es mi intención entrar ahora en discusión acerca de si en Aristóteles pudiera encontrarse implícita o explícita una cierta idea de la creación. Me bastará referiume aquí a quienes han estudiado este punto. Véase, por ejemplo, la conclusión del ya citado J. M. Laso: "Las ideas de providencia y de creación son totalmente ajenas a este esquema teológico de Aristóteles. Sin embargo, y a pesar de todo esto, la existencia de este Dios es

\footnotetext{
15. MAYOR, D., Problemas sobre Anaxágoras, en Pensamlento (fulio-agosto-septiembre) (1954)

16. LASO GONZALEZ, J. M. Dos concepciones det motor inmóvil incompatibles entre sI: Iib. VIII de la Physlca y el lib. XII de los Metaphysica de Aristóteles, en Revista de Filosofía, pág 379-414.

17. VUILLEMIN, J., De la Logique a la Théologle, Parls 1967

18. "Motor que existe él mismo como ser inmóvil, ser en acto, éste no puede de ninguna manera cambiar... es un ser necesario... y en el orden de su necesidad es también un ser de orden de lo bello... principio de oue depende el cielo y la Naturaleza toda. La duración y la vi$\mathrm{da}$, tan breves pora nosotros. las posee este ser en el meior grado, poraue él siempre es aqueIlo, cosa para nosotros imposible, y porque también el placer es su acto... El pensamiento en si es el pensamiento de lo que es mejor, y el pensamiento por. excelencia es el de lo que es bueno por excelencia. Se entiende a si mismo abarcandolo inteligible... de manera que lo inteligible y el entendimiento llegan a ser lo mismo...; la actuación de la inteligencia es la posesión de lo inteligible..., esto más que otra cosa parece ser ese algo divino que posee la inteligencia, y la contemplación es el placer supremo y el bien absoluto. Si el ser divino se halla siempre en esta felicidad que nosotros gozamos breves instantes, es bien digno de admiración, y si su felicidad es aún mayor, es con más razón aún merecedor de nuestra admiración. $Y$ seguramente así es... Es también vida... porque la inteligencia es una vida, y esta actualidad, tomada en si misma, es su vida perfecta y eterna. Decimos que el ser divino es un ser animado, eterno y perfecto. De manera que la vida, la duración continua y eterna pertenecen al ser divino. Esto es, en efecto, el ser divino". Met, XII, 7.
} 
demostrada por Aristóteles. En resumidas cuentas, Aristóteles encontró necesaria la existencia de un Dios perfecto, al considerar el orden del Cosmos y la gradación ontológica de los seres que conoce el hombre como buenos en sí mismos; pero tras delimitar la naturaleza del ser óptimo, no encontró la forma adecuada para explicar la realidad cambiante partiendo de él, sin que despreciara su plenitud de sers) ${ }^{19}$. Es decir, si bien el movimiento de los seres físicos de la creación tendrá una explicación original adecuada al decir Aristóteles que son movidos por Dios como objeto de conocimiento y de deseo dependiendo de una potencia eterna y única, esta dependencia no afecta a la posición misma del ser. O lo que es lo mismo, Aristóteles no habría sido capaz de eludir el dualismo ${ }^{20}$.

No cabe duda que este esfuerzo de la razón humana que se remonta a làs alturas del ser supremo es algo grande, pero en estos casos y en los demás que pudieran aducirse de la historia de la filosofía este sor trascendente se encuentra siempre enfrentado con un mundo compuesto en todo o en parte por una materia eterna. Y es entonces cuando, al comparar esta concepción del Dios trascendente a la materia, enfrentada siempre consigo, con un panteísmo de tipo hegeliano, no sabe uno a qué carta quedarse como explicación menos inadecuada de la realidad de las relaciones entre Dios y el mundo. Por un lado la trascendencia es sublime, pero se viene abajo a causa de la eternidad de la materia puesta en frente; por otro, la suprema razón de ser de todo en el panteísmo queda oscurecida por la imperfección ontológica y radical de ese ser supremo que necesita una evolución interna para llegar a ser perfecto, pues es elemental que, teniendo el ser inicial toda la perfección - pues toda de él procede por emanación- y de una manera absoluta, o sea, sin relación a otro alguno, la tenga también ya desde el principio de un modo perfecto. En una palabra, a pesar de todo el resultado positivo que podemos atribuir a la mente humana en este esfuerzo en la búsqueda de Dios, hay que reconocer que de hecho no ha llegado a esa trascendencia absoluta que aluda a un Dios personal como el que se supone en el que es principio del mundo por creación del mismo de la nada, y que es el que se señala como término de las vías.

\footnotetext{
19. LASO GONZALEZ, J. M., Análisis de las aporías teológlcas del I'bro XII de los "Metaphysica" de Aristóteles, en Naturaleza y Gracia (enero-abril) 1969, pp. 3-36.

20. Véase una detallada exposición del tema de la creación en la filosof́a griega en MANSER, G. M., La esencla del tomismo, Madrid, 1947, pp. 610-628.
} 
LA CUESTION (DE IURE),

La cuestión "de facto» está clara; la filosofía no cristiana y puramente racional no llega a la trascendencia absoluta. Pero, ¿es que es sólo cuestión "de facto» o también (de iure»? Refiriéndonos en concreto a Aristóteles, y en él a todos los demás, podríamos preguntarnos: cacaso Aristóteles solamente no supo llegar de hecho a un Dios trascendental como creador o más bién no pudo llegar? Esta pregunta nos coloca frente a frente a lo que, según mi modesto parecer, es la dificultad fundamental y única contra la validez puramente racional de las clásicas vías. Hirschberger, a propósito de la dificultad de las vías a causa del raciocinio en ellas empleado, responde que (se puede decir que, en efecto, de hecho el raciocinio es difícil, pero ciertamente no tan extremadamente difícil como se propugna) ${ }^{21}$. Ciertamente así es si la dificultad se limita al aspecto metafísico en que se desarrolla el raciocinio, aspecto para el que hay que estar suficientemente preparado. Pero la dificultad no está solamente ahí, está en algo más profundo que es la relación íntima existente entre Dios y la realidad mundana que ha de ser descubierta por una razón dejada a sus solas fuerzas, no impregnada como está la de un cristiano por la revelación. Aquí está el verdadero (quid)" de la dificultad que, modestamente hablando, me parece más difícil y extremadamente más difícil de como se presenta ordinariamente la dificultad del raciocinio en las pruebas de la existencia de Dios. En efecto, el salto entre el (secundus gressus) de las célebres vías y el término de las mismas : motor inmóvil, causa primera incausada, ser necesario, etc. absolutamente trascendentes, es verdaderamente abismal, un salto hacia las tinieblas, una especie de salto en el vacío. Entiéndase bien, hablamos de un salto en el vacío desde un punto de vista subjetivo, lo cual no quiere decir que ontológicamente la contingencia radical de los seres, su extrema y absoluta indigencia óntica, no lleve consigo la exigencia de la trascendencia absoluta. Pero una cosa es lo que la realidad es en sí misma y otra lo que nosotros podemos alcanzar acerca de ella. Repetimos que la dificultad para salvar ese abismo tenebroso entre los seres y el SER es de tipo subjetivo-objetivo: es cuestión de la comprensibilidad, por parte del que conoce, de la realidad que tiene delante de sí; es decir, sólo podemos salvar subjetivamente ese abismo si llegamos a una noción adecuada de la

21. HIRSCHERGER, J., o. c., p. 112. 
trascendencia del ser necesario, si la relación entre el SER y los seres que, como trascendente incluye la noción de creación, está al alcance de la mente de modo que por sí misma puede sospecharla y planteársela como hipótesis. Ahora bien, contra la posibilidad de poder llegar a esta hipótesis está lo siguiente: la acción creadora es por una parte acción exclusiva de Dios y en ninguna parte podría ser experimentada o conocida por nosotros sino en El, lo cual es imposible; por otra parte, el no-ser de las creaturas, sobre todo si insistimos en aquello de que "omnis cognitio incipit a sensu», lo experimentamos únicamente como una variante del ser total. Ahí tenemos todo ese esfuerzo de las filosofías panteístas incapaces de establecer la personalidad del hombre frente al ser supremo por el cual, tarde o temprano, termina por ser absorbido. La historia de esta despersonalización del hombre es tarantigua y tan moderna como el mismo pensamiento filosófico una . que se ha independizado de los mitos de la antigüedad o cuando no ha sabido captar el mensaje de la revelación. Y esto es muy natural, ya que, por otra parte, en una trascendencia absoluta, el ser existencial de la creatura es otro de Dios, pero a la vez es nada fuera de Dios (trascendencia-inmanencia). Es decir, Dios que, como creador, es trascendente en un sentido estricto, tiene que ser inmanente y con una inmanencia total dada la inconsistencia absolutamente radical en el aspecto óntico de la creatura. ¿Quién será capaz de comprender este ser-no-ser de la realidad? Recuérdese que Kant encontró aquí una de sus antinomias. ¿No pecaríamos de ingenuos si nos empeñásemos en considerar desvelada a la simple razón la misteriosa relación Dios-creatura en su aspecto de creador? ${ }^{22}$. La creatura es y puede dejar de ser sin la más mínima mutación por parte de la esencia divina y de su voluntad que. sin embargo, han de serle inmanentes ${ }^{23}$. ¿Qué es, pues, esta creatura? No diremos que sea un absurdo, pero sí que es algo incomprensible para la pura razón humana a la hora de reducir la multiplicidad a la unidad, lo contigente a lo necesario. La razón pura ha podido darnos solamente un Acto puro aristotélico, grandioso por cierto en su descripción como ya hemos visto, y también un dios hegeliano no menos sublime en su realización total después de su encarnación en la tragedia

\footnotetext{
22. No se olvide que a Dios le ha costado lo suyo hacer comprender a los hombres la revelación de su trascendencia, revelación que ha seguido un "largo proceso, jalonado por una serie de intervenciones proféticas, (que) desemboca con el segundo Isaias en una especie de Evangelio de la trascendencia divina" (V. LARCHER, CH., La trascendencia divina, otra causa de la ausencia de Dios, en CONCILIUM, dic. 1969.

23. Dios es "intimior intimo meo, superior summo meo". Conf. III, 11.
} 
de lo finito. Y ciertamente habría que decir que no hubo de ser pequeña la tarea y el esfuerzo para concebir a un dios como "Espíritu del mundo (y capaz de) deshacerse de toda esencia objetiva extraña y comprenderse por fin como espíritu absoluto, engendrando de si mismo lo que es objetivo para él reteniéndolo tranquilamente en su poder ${ }^{24}$. En realidad, ¿a qué más podría haber aspirado la razón humana en sus atisbos acerca del creador? Aquí está, y solamente aquí, la gran dificultad contra la posibilidad de la demostración racional metafísica de la existencia de Dios partiendo de la realidad física de este mundo. ¿Cómo podría, pues, concluir a esa existencia trascendente si la creación, de la que tiene que hechar mano necesariamente para dar el salto, es incomprensible? Tanto esto es así que, aun admitido por la revelación el hecho de la creación y elaborada filosóficamente su noción, podremos dar ciertamente una explicación más adecuada de la relación Dios-creatura evitando las grandes dificultades y absurdos de dualismos y panteísmos, pero no será menos cierto que cuando el panteísta nos pida una explicación que desvele el misterio de una realidad que puede ser y no ser, comenzar y desaparecer sin la más mínima mutäción o cambio de la realidad divina, tendremos que refugiarnos en el misterio. Podríamos responderle únicamente que la incomprensibilidad de una solución no es un absurdo y no obsta para que pueda ser entendida como más aceptable para resolver el problema. Teniendo todo esto en cuenta me parece podemos comprender mejor, sin recurrir a otras componendas, el que Âristóteles llegue a esa conclusión genial del Acto puro que, sin embargo, no lo sea tanto al tener que admitir un dualismo por razón de la materia.

\section{CONCLiUsion}

Aplicando a las pruebas tradicionales lo expuesto anteriormente, podemos concretar los puntos siguientes:

I) Creemos que la noción filosófica de la creación es necesaria para dar legítima y científicamente el salto desde las creaturas hasta la trascendencia de un primer principio al que podamos aplicar con toda propiedad aquel (hoc dicimus Deum»).

24. FloREZ, R., El Dios dialéctico, en Estudio agustiniano. Enero-abril, 1970, p. 48. 
2) Históricamente tenemos esta noción (nos referimos al contenido, no a la noción filosófica) por revelación, y fuera de ella no se encuentra.

3) Nos parece, salvo siempre mejor opinión, que dicha noción (sin la revelación del hecho de la creación) supera las fuerzas de la pura razón natural tal como se encuentra ésta en sus relaciones con un Dios al que haya de dirigirse a través de las cosas creadas; y la razón es que la noción de creación, que necesariamente está implicada en la demostración racional de que aquí se trata, es algo misterioso y su comprensión exigiría el conocimiento del acto creativo en sí mismo. Ahora bien, no es lícito suponer una noción, que es ingrediente necesario para una explicación, si esa noción es incomprensible: "las razones no tienen más remedio que moverse dentro del campo inteligible) ${ }^{25}$. En una palabra, (nada de metafísica del ser puro sin la revelación bíblica) ${ }^{26}$.

4) Una vez revelado el hecho de la creación, la razón humana ha elaborado no sin grandes esfuerzos la noción filosófica de la misma. $Y$ es entonces cuando se encuentra ya capacitada para dar una explicación de las relaciones existentes entre el Ser necesario y el ser radicalmente contigente. $Y$ aunque en sí misma la creación permanezca envuelta en el misterio, al ser una realidad su traducción a fórmula filosófica, esta noción es una explicación racional de dichas relaciones e ilumina a la mente para que pueda evitar el absurdo de una evolución panteísta o de una eternidad de los dos seres: Dios-materia.

5) Si se nos dice que entonces las vías, como demostración racional, son válidas solamente para aquel que admita la revelación, le diremos que, sin duda, para este tal valen, pero no porque ese hecho de la revelación sea puesto como premisa lógica de la demos. tración. Es decir, cuando se trate de un no cristiano habría que

25. KIERKEGAARD, S., La enfermedad mortal, o de la desesperación y el pecado, Madrid 1969 , p. 196.

V. MANSER, G. M., La esencla del tomismo, Madrid 1947, pp. 638 ss. Manser, al tratar la cuestión de la posibilidad de una demostración rigurosa de la creación en favor de la que estaría Santo Tomás en contra de San Alberto, se pronuncia en contra de dicha posibilidad diciendo: "Para $\mathrm{mi}$ es muy significativo que ni Alberto Magno ni Maimónides, que, sin embarço, eran cerebros poderosos considerarán la creación de la nada como filosóficamente demostrable". Por nuestra parte creemos que habrí que hacer una distinción que más bien daría la razón a ambas partes; sería ésta: si se trata de una demostración racional, dejada la razón a sus solas fuerzas, hay que' estar con San Alberto. Si tenemos en cuenta que Santo Tomás ya tiene la noción de creación elaborada a base del hecho de la fe, la posibilidad de la demostración de la existencia de Dios puede también defenderse, ya que la realidad de la creación está entrañada en la potencialidad absolutamente radical y radicalmente absoluta del ser contingente.

26. GILSON, E., Lo spirito della filosofia medievale, Brescia 1947, p. 38 ss. 
presentarle no el hecho de la creación revelado por la fe, sino $\mathrm{h}$ noción filosófica de creación ya elaborada con la ayuda de la filosofía griega y que ya poseemos; se le invitaría a examinar esta noción filosófica de creación como un medio de relacionar al ser supremo y primer principio de todas las cosas con las cosas existentes, medio que parece dejar mejor a salvo los derechos del ser supremo y que evita mejor las contradicciones y absurdos, o al menos, la incomprensibilidad de un dualismo y a fortiori de un panteísmo evolucionista; aunque, naturalmente, también de nuestra parte exista la oscuridad, no absurdo, en la comprensión del hecho de la creación que, como queda dicho nos hubiera impedido sospechar la posibilidad de esa noción. Es decir, con la ayuda de la filosofía griega tendríamos de hecho una metafísica originada en la revelación pero que ya no sería revelación ni habría por qué presentársela así al incrédulo. Precisamente tendríamos aquí un reforzamiento interno de la filosofía por parte de la revelación que enriquece a la filosofía, un válido apoyo prestado a ésta para desentrañar todo el significado de las nociones de contigencia y acto puro que para la filosofía eran una verdadera exigencia (recuérdese todo el empeño de Aristóteles parn purificar al primer motor a pesar del dualismo), pero algo muy misterioso que impedía a la pura razón adentrarse en lo más profundo de dichas nociones metafísicas. Son de G. Marcel estas palabras: "Si l'exigence de transcendance se présente avant tout comme insatisfaction, (sependant) la réciproque ne paraît vrai, il ne semble pas qu'on soit en droit de dire que toute insatisfaction implique l'aspiration à la transcendances) ${ }^{27}$.

En una palabra, si las vías, como demostración puramente racional tienen sus más menos que más en orden a la validez, sin embargo, con la ayuda de la revelación, en el sentido expuesto en estas váginas. pueden ser usadas honradamente para una afirmación filosó ica válida de la existencia de Dios.

\section{EPILOGO}

La exposición que precede nos hace comprender mejor la necesi. dad de la revelación que, en este caso, si bien no es necesaria para que

27. MARCEL, G., Le mystère de l'être, t. I, Réflexion et mystêre (1951), p. 50. 
la razón se remonte a un principio de todas las cosas en un sentido corriente, hacia el que deba sentir una responsabilidad (muy bien lo de la Sabiduría condenando a los filósofos antiguos), sí que parece ser necesaria absolutamente para una comprensión intelectual de la trascendencia personal y científicamente filosófica de ese primer principio $y$, por consiguiente, para una validez absoluta de las vías. Se verificaría aquí también aquello de que "las causas son causas recíprocamente». Sin la revelación de la trascendentalidad absoluta de Dios, la razón no podría llegar a esa trascendencia absoluta; pero sin la filosofía, el hombre, aceptando el hecho sin más de la creación, no podría en el campo de la reflexión humana hacer ver al panteísta un camino hacia ese primer principio que salve mejor la naturaleza de éste, sus relaciones con lo que de él ha salido y que evite los absurdos propios de un evolucionismo absoluto. Citando de nuevo a Gilson y completando la frase antes citada: "Nada de metafísica del ser puro, sin la revelación bíblica; pero nada tampoco de metafísica salida de la revelación, sin la filosofía griegas ${ }^{28}$. Reforzamiento de la filosofía con la fe; ¿filosofía cristiana? ${ }^{29}$.

Fidel Casado

28. GILSON, E., O. C.
29. LRESMONTANT, C. Lorlg ne della fllosofla cristlana, ed. Paoline, Catania 1963. 